\title{
GC-MS/FID analysis of aldehyde and hydrocarbon emissions from exhaust gases of a spark ignition engine fueled with different ethanol-gasoline blends
}

\begin{abstract}
In this paper the influence of changes in ethanol concentration in gasoline-alcohol blend on the concentrations of aldehydes and hydrocarbons emitted from a spark-ignition automotive engine is described. Results of the exhaust gas analysis with E0, E5, E10, E25 and E50 blends tested in a car meeting the Euro 5 emission standard are presented. It was found that an increase of the ethanol concentration in the fuel blend leads to an increase in the acetaldehyde concentration in the exhaust gas. TD-GC-FID/MS analysis of exhaust gases collected on two different, complementary sorbents (Carbograph 1TD, Tenax TA) allowed for identification of the large number of hydrocarbons.
\end{abstract}

Key words: gasoline-ethanol blends, aldehydes, hydrocarbons, exhaust gas analysis

\section{Analiza emisji aldehydów i węglowodorów w spalinach silnika z zapłonem iskrowym zasilanego różny- mi mieszankami benzyny z etanolem metodą GC-MS/FID}

\begin{abstract}
W artykule omówiono wpływ zmiany stężenia etanolu w mieszance benzyna-alkohol na zawartość aldehydów $i$ węglowodorów w spalinach silników samochodowych o zapłonie iskrowym. Przedstawiono wyniki analiz spalin z mieszanek E0, E5, E10, E25 i E50 prowadzonych dla samochodu spetniajacego wymagania dotyczace emisji Euro 5. Zaobserwowano, że wraz ze wzrostem stężenia etanolu w mieszance paliwowej zwiększa się stężenie acetaldehydu w spalinach. Analiza $z$ wykorzystaniem technik TD-GC-FID/MS spalin pobranych na dwa różne, uzupetniające się sorbenty (Carbograph ITD, Tenax TA) pozwolita na identyfikację dużej liczby węglowodorów.
\end{abstract}

Słowa kluczowe: mieszanki benzyna-etanol, aldehydy, węglowodory, analiza spalin

\section{Introduction}

Hundreds of organic and inorganic compounds, many of which have a negative influence on the environment and human health, are created during the combustion of fuel in the engine. These include, among others, oxides of nitrogen $\left(\mathrm{NO}_{\mathrm{x}}\right)$, carbon monoxide $(\mathrm{CO})$, and hydrocarbons $(\mathrm{HC})$, including aromatic hydrocarbons and aldehydes. Aromatic hydrocarbons such as benzene and napthalene are considered carcinogenic. Furthermore, carbonyl compounds such as formaldehyde, acetaldehyde, acrolein and methyl ethyl ketone (MEK) are classified as irritants, mutagens and / or carcinogens [1]. Emission standards (called the EURO standards 1 to 6) enforce measures to reduce their quantity and toxicity. The current (as of 1 September 2009), Euro 5 standard [2] specifies, for new cars with gasoline engines, the maximum $\mathrm{CO}$ emission at the level of $1000 \mathrm{mg} / \mathrm{km}$, non-methane hydrocarbon emission of $68 \mathrm{mg} / \mathrm{km}$, total hydrocarbon emissions of $100 \mathrm{mg} / \mathrm{km}, \mathrm{NO}_{\mathrm{x}} 60 \mathrm{mg} / \mathrm{km}$ (i.e. a 25\% reduction compared with the Euro 4 standard), and particulate matter (for engines with direct injection) at $5 \mathrm{mg} / \mathrm{km}$. From the introduction of the Euro 6 standard, planned for 2014 , the requirements for emissions of pollutants from new cars will increase, including the introduction of a particle number emission limit for SI vehicles featuring direct injection engines. While the toxic nature of aldehyde emissions is widely recognized, no emissions limits are planned for the EU (currently, only the Californian legislation 'California Code of Regulations' covers aldehyde emissions). However,

\section{Wstęp}

Podczas spalania paliwa w silniku spalinowym powstaje kilkaset związków organicznych i nieorganicznych, z których wiele ma negatywny wpływ na środowisko i zdrowie człowieka. Można do nich zaliczyć między innymi tlenki azotu $\left(\mathrm{NO}_{\mathrm{x}}\right)$, tlenek węgla $(\mathrm{CO})$, węglowodory $(\mathrm{HC})$, w tym węglowodory aromatyczne i aldehydy. Węglowodory aromatyczne, takie jak np. benzen, naftalen uznawane są za rakotwórcze. Również związki karbonylowe, takie jak formaldehyd, acetaldehyd, acroleina, metyloetyloketon (MEK) zalicza się do drażniących, mutagennych i/lub rakotwórczych [1]. Standardy emisji spalin (określane jako normy Euro od 1 do 6) wymuszają działania mające na celu ograniczenie emisji związków szkodliwych spalin. Obecnie obowiązująca (od 1 września 2009 r.) norma Euro 5 [2] określa, dla nowych samochodów z silnikiem benzynowym, maksymalną emisję CO o wartości $1000 \mathrm{mg} / \mathrm{km}$, węglowodorów niemetanowych $68 \mathrm{mg} / \mathrm{km}$, całkowitej emisji węglowodorów $100 \mathrm{mg} / \mathrm{km}$, $\mathrm{NO}_{x} 60 \mathrm{mg} / \mathrm{km}$ (co stanowi 25-procentową redukcję w porównaniu z normą Euro 4), a cząstek stałych (tylko dla samochodów z wtryskiem bezpośrednim benzyny) $5 \mathrm{mg} / \mathrm{km}$.

Od roku 2014 planowane jest wprowadzenie normy Euro 6 zaostrzającej wymagania dotyczące emisji substancji szkodliwych przez nowe samochody, w tym wprowadzenie limitów emisji cząstek stałych przez samochody z silnikami o bezpośrednim wtrysku benzyny. Aldehydy, pomimo że jest znane ich toksyczne działanie, nie są objęte jeszcze limitami emisji w przepisach europejskich. Jedynie wymagania 
ongoing research in the European Union and the United States define these compounds as expected to be regulated under future emissions regulations (e.g. Euro 7), along with other compounds, to reduce emissions of exhaust gases of internal combustion engines such as $\mathrm{NO}_{2}, \mathrm{~N}_{2} \mathrm{O}, \mathrm{NH}_{3}$.

One of the potential ways to reduce emissions of harmful compounds in vehicle exhaust is the use of alternative fuels, or fuels with the addition of bio-components. Alcohols such as methanol $(\mathrm{MeOH})$ and ethanol $(\mathrm{EtOH})$ are added as oxidisers (low concentrations of alcohol) or alternative fuels (mixtures with high concentration of alcohol). Ethanol has more favourable properties than methanol as it exhibits greater stability and tolerance to water (lowering the risk of corrosion of fuel system components), and has much lower toxicity [3]. The results of emission testing of gasoline-alcohol blends have demonstrated that compared to "traditional" gasoline, increasing the concentration of alcohol in the blend leads to reductions in emissions of $\mathrm{CO}$ and hydrocarbons (including aromatics), whereas emissions of aldehydes increase [3 - 7]. $\mathrm{NO}_{\mathrm{x}}$ emission is variable, but generally the addition of ethanol increases these emissions [4].

This study focuses primarily on determining the impact of increased ethanol content in the fuel on the emission of aldehydes, mainly acetaldehyde resulting from the incomplete oxidation of ethanol. The acetaldehyde content in the exhaust gas was determined as a derivative 2-hydroxymethylpiperidine (2-HMP) by GC-MS/FID gas chromatography.

Hydrocarbons present in the exhaust gas were also identified. Samples were collected by an active method on two different sorbents, Carbograph 1TD and Tenax TA, and then subjected to thermal desorption and analyzed using gas chromatography coupled with mass spectrometry.

\section{Experimental}

\subsection{Samples}

The exhaust gases analyzed resulted from combustion of gasolineethanol blends containing varying ethanol contents: $0 \%, 5 \%, 10 \%, 25 \%$ and $50 \%$ (by volume). The exhaust gases were generated by a passenger car with a spark ignition engine of cylinder displacement approximately $1.3 \mathrm{dm}^{3}$, with 8 valves and a closecoupled TWC aftertreatment system, which fully met all requirements of the relevant Euro 5 standard. Tests were carried out in the exhaust emission laboratory at BOSMAL Automotive Research and Development Institute, described in [8] and shown in Figure 1, based on the New European Driving Cycle (NEDC) [9] (Fig. 2). This cycle is a commonly used driving cycle for quantifying emissions of harmful exhaust gas kalifornijskie (California Code of Regulation) w USA określają obecnie limity emisji aldehydów. Jednak prowadzone obecnie prace badawcze w krajach Unii Europejskiej i USA określają te związki jako planowane do limitowania ich emisji w przyszłych przepisach Euro 7, obok innych związków do ograniczania w spalinach silników spalinowych, jak: $\mathrm{NO}_{2}, \mathrm{~N}_{2} \mathrm{O}, \mathrm{NH}_{3}$.

Jednym z potencjalnych sposobów ograniczenia emisji związków szkodliwych spalin jest stosowanie paliw alternatywnych bądź paliw z dodatkiem biokomponentów. Alkohole, takie jak metanol $(\mathrm{MeOH}) \mathrm{i}$ etanol $(\mathrm{EtOH})$ dodawane są do paliwa jako dodatki utleniające (małe stężenie alkoholu) lub alternatywne paliwa (mieszanki o dużym stężeniu alkoholu). Etanol, z uwagi na lepszą stabilność i tolerancję wody, mniejsze ryzyko korozji elementów układu paliwowego i znacznie mniejszą toksyczność, ma lepsze właściwości od metanolu [3]. Badania emisji spalin z mieszanek benzyna-alkohol wskazują, że w porównaniu z tradycyjną benzyną na ogół ze wzrostem stężenia alkoholu w mieszance następuje zmniejszenie emisji $\mathrm{CO}$ i HC, w tym aromatycznych, natomiast zwiększa się emisja aldehydów [3 - 7]. Emisja $\mathrm{NO}_{\mathrm{x}}$ jest zmienna, ale generalnie dodatek etanolu zwiększa ich emisję [4].

$\mathrm{W}$ badaniach przedstawionych $\mathrm{w}$ tej pracy skupiono się głównie na określeniu wpływu wzrostu zawartości etanolu w paliwie na emisję aldehydów, głównie acetaldehydu powstającego w wyniku niepełnego utlenienia etanolu. Zawartość acetaldehydu w spalinach oznaczano techniką chromatografii gazowej GC-MS/FID jako pochodną 2-hydroksymetylopiperydyny (2-HMP).

Podjęto również próbę identyfikacji węglowodorów obecnych w spalinach. Próbki pobierano metodą aktywną 


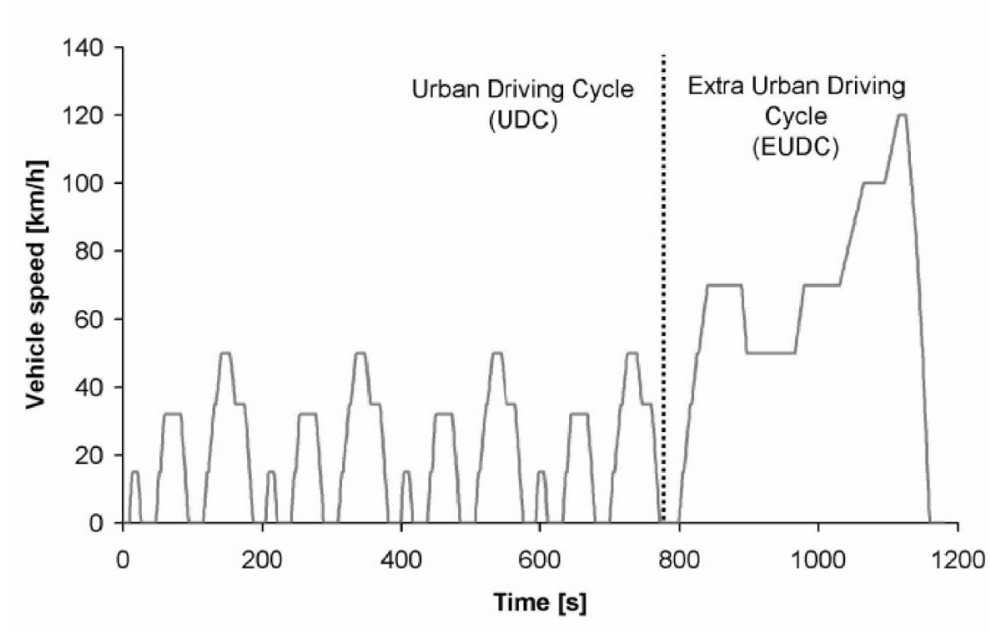

Fig. 2. New European Driving Cycle (NEDC) Rys. 2. Nowy Europejski Cykl Jezdny (NEDC)

components on a chassis dynamometer, with a cold start and two phases: urban driving conditions (UDC), followed by extra-urban driving conditions (EUDC), covering a total distance of approximately $11 \mathrm{~km}$.

Before the tests, the fuel blends were initially analyzed using the chromatography method in order to confirm the same gasoline base. Due to the fact that standard gasoline available at filling stations in the EU contains approximately $5 \%$ ethanol, E5 blend was used as the base fuel.

\subsection{Sampling}

The exhaust gases were collected by a pump (flow rate 6 $\mathrm{dm} 3 / \mathrm{min}$ ) into a Tedlar bag (of approximate volume $150 \mathrm{dm}^{3}$ ), directly from the exhaust, over the entire driving cycle. During the test, $5547 \mathrm{dm}^{3}$ of exhaust gases were generated, and the theoretical distance traveled by the vehicle was $11.007 \mathrm{~km}$.

The exhaust gases were collected from the tedlar bag onto suitable sorbent tube. The parameters of the sampling are presented in Table 1.

In the case of sampling onto Tenax TA and 2-HMP tube, pre-filters with sodium thiosulfate $\mathrm{Na}_{2} \mathrm{~S}_{2} \mathrm{O}_{3}$ and potassium iodide KI were used. Their role is to reduce the negative impact of nitrogen oxides and ozone (both of which are present in the exhaust gas) on the sorbents.

\subsection{Chromatography analysis}

Chromatography analysis was performed with the use of an Agilent 7890 gas chromatograph (GC) with simultaneous detection (splitter) on the flame ionization detector (FID) and mass detector (MSD, Agilent 5975C) (Fig. 3). na dwa różne sorbenty, Carbograph 1TD oraz Tenax TA, a następnie poddawano termodesorpcji i analizowano z wykorzystaniem techniki chromatografii gazowej sprzężonej ze spektrometrią masową.

\section{Badania wlasne}

\subsection{Próbki badawcze}

Spaliny poddawane badaniom były produktem spalania mieszanek benzyna-etanol o różnej zawartości alkoholu: 0\%, 5\%, 10\%, 25\% i 50\%. Spaliny pochodziły z samochodu osobowego z silnikiem ZI, o objętości skokowej około 1,3 dm³, 8-zaworowym, wyposażonym w reaktor katalityczny, spełniającego wymagania normy emisji Euro 5. Testy prowadzono w laboratorium badań emisji spalin Instytutu BOSMAL, opisanym w pracy [8] i przedstawionym na rys. 1, gdzie wykorzystano nowy europejski test jezdny (NEDC) [9] (rys. 2). Jest to powszechnie stosowany cykl jezdny w badaniach emisji związków szkodliwych spalin, wykonywany na hamowni podwoziowej, cechujący się zimnym rozruchem oraz miejskimi i pozamiejskimi warunkami jazdy, o łącznym dystansie wynoszącym około $11 \mathrm{~km}$.

Przed przystąpieniem do badań mieszanki paliw poddano wstępnej analizie chromatograficznej mającej na celu potwierdzenie jednakowej bazy benzynowej. Ponieważ tradycyjna benzyna dostępna na stacjach benzynowych zawiera około 5\% etanolu, paliwo E5 potraktowano jako bazowe.

\subsection{Pobór próbek}

Spaliny pobierano za pomocą pompy (przepływ $6 \mathrm{dm}^{3} / \mathrm{min}$ ) do worka tedlarowego o pojemności około $150 \mathrm{dm}^{3}$, bezpośrednio z układu wylotowego samochodu, w czasie trwania całego testu NEDC. Podczas testu generowane było $5547 \mathrm{dm}^{3}$ spalin, a teoretyczna droga przebyta przez samochód wynosiła 11,007 km.

Z worka tedlarowego spaliny pobierano na odpowiednie rurki sorbentowe. Parametry poboru próbek przedstawiono w tabeli 1.

W sytuacji poboru próbek na rurki z Tenax TA oraz 2-HMP zastosowano filtry wstępne $\mathrm{z}$ tiosiarczanem sodu $\mathrm{Na}_{2} \mathrm{~S}_{2} \mathrm{O}_{3}$ i jodkiem potasu KI. Mają one zadanie ograniczenia negatywnego wpływu na sorbenty obecnych w spalinach tlenków azotu i ozonu.

\subsection{Analiza chromatograficzna}

Analizę chromatograficzną prowadzono z wykorzystaniem chromatografu gazowego Agilent 7890A (GC) z

Table 1. Parameters of exhaust gas sampling

Tabela 1. Parametry poboru próbek spalin

\begin{tabular}{|c|c|c|c|}
\hline Compound group/grupa związów & Sorbent/sorbent & $\begin{array}{l}\text { Flow rate/przeptyw } \\
\qquad[\mathrm{ml} / \mathrm{min}]\end{array}$ & $\begin{array}{c}\text { Sample volume/ } \\
\text { objętość próbki } \\
{\left[\mathrm{dm}^{3}\right]}\end{array}$ \\
\hline \multirow{2}{*}{$\begin{array}{l}\text { Aromatic and aliphatic hydrocarbons/ } \\
\text { weglowodory aromatyczne i alifatyczne }\end{array}$} & Carbograph 1TD/carbograph 1TD & 100 & 1 \\
\hline & Tenax TA/Tenax TA & 100 & 1 \\
\hline Aldehydes/aldehydy & $\begin{array}{l}\text { 2-hydoxymethylpiperidine (2-HMP) on XAD-2 resin/ } \\
\text { 2-hydroksymetylopiperydyna (2-HMP) na złożu XAD-2 }\end{array}$ & 100 & 3 \\
\hline
\end{tabular}




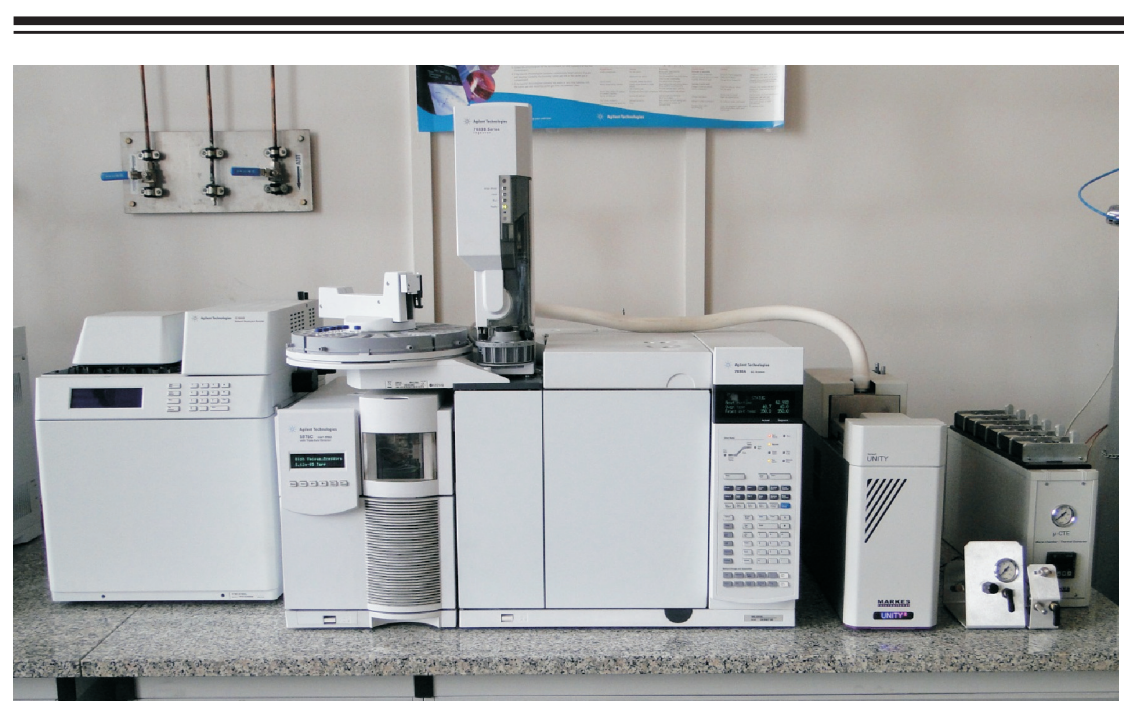

Fig. 3. Chromatography system: GC Agilent 7890A with autosampler 7683B, splitter and FID detector, MSD Agilent 5975C mass selective detector, Agilent G1888 headspace sampler, Markes UNITY2 thermodesorber and Markes $\mu$-CTE (Micro-Chamber Thermal Extractor), situated within BOSMAL's chemical laboratory

Rys. 3. Zestaw chromatograficzny: GC Agilent 7890 A z podajnikiem 7683 B, dzielnikiem strumienia gazu i detektorem FID, MSD Agilent 5975C, próbnikiem headspace Agilent G1888, termodesorberem Markes UNITY2 i mikrokomora Markes $\mu$-CTE (Micro-Chamber Thermal Extractor) w laboratorium chemicznym w BOSMAL jednoczesną detekcją (dzielnik strumienia gazu) detektorem płomieniowojonizacyjnym (FID) i masowym (MSD, Agilent 5975C) (rys. 3).

W analizie węglowodorów rurki sorbentowe poddawano desorpcji termicznej (termodesorber Markes UNITY2, pułapka wymrażająca U-T11GPC-2S general purpose carbon $(\mathrm{C} 4 / 5-\mathrm{C} 30 / 32))$ przez 15 min w temperaturze $300{ }^{\circ} \mathrm{C}$, a następnie wprowadzano na kolumnę chromatograficzną ze splitem 30:1. Rurki sorbentowe do analiz aldehydów poddawano ekstrakcji $1 \mathrm{ml}$ toluenu (z dodatkiem dimetyloformamidu (DMF) jako wzorca wewnętrznego, $20 \mu \mathrm{l}$ $\mathrm{DMF} / 100 \mathrm{ml}$ toluenu) w łaźni ultradźwiękowej przez $30 \mathrm{~min}$. Warunki analiz chromatograficznych przedstawiono $\mathrm{w}$ tabeli 2.

Identyfikację związków prowadzono, opierając się na chromatogramie uzyskanym z detektora masowego.

Table 2. Parameters of chromatography analysis

Tabela 2. Warunki analizy chromatograficznej

\begin{tabular}{|c|c|c|c|}
\hline \multirow[t]{3}{*}{ Parameter/parametr } & \multicolumn{3}{|c|}{ Analysis parameters/warunki analizy } \\
\hline & \multirow{2}{*}{$\begin{array}{l}\text { gasoline-ethanol blend/paliwo ben- } \\
\text { zyna-etanol }\end{array}$} & \multicolumn{2}{|c|}{ exhaust gases/spaliny } \\
\hline & & Hydrocarbons/węglowodory & Aldehydes/aldehydy \\
\hline $\begin{array}{l}\text { Chromatography column/ } \\
\text { kolumna chromatograficzna }\end{array}$ & $\begin{array}{l}\text { DB-5ms UI (Agilent); } \\
60 \mathrm{~m} \times 320 \mu \mathrm{m} \times 1 \mu \mathrm{m}\end{array}$ & $\begin{array}{l}\text { DB-5ms UI (Agilent); } \\
60 \mathrm{~m} \times 320 \mu \mathrm{m} \times 1 \mu \mathrm{m}\end{array}$ & $\begin{array}{c}\text { DB-1301 (Agilent); } \\
30 \mathrm{~m} \times 320 \mu \mathrm{m} \times 1 \mu \mathrm{m}\end{array}$ \\
\hline Injector/dozownik & $\begin{array}{c}\text { injection } 1 \mu \mathrm{l} \text {, split } 200: 1 \text {, } \\
\mathrm{t}=300{ }^{\circ} \mathrm{C} \text {, carrier gas } \mathrm{He} \text {, constant } \\
\text { flow } 2,5 \mathrm{ml} / \mathrm{min} / \text { nastrzyk } 1 \mu \mathrm{L}, \\
\text { podziat } 200: 1, t=300^{\circ} \mathrm{C} \text {, gaz nośny } \\
\text { He, staty przeptyw } 2,5 \mathrm{ml} / \mathrm{min}\end{array}$ & $\begin{array}{l}\text { splitless mode, } \mathrm{t}=300{ }^{\circ} \mathrm{C} \text {, carrier } \\
\text { gas He, constant pressure } 23 \mathrm{psi} / \\
\text { tryb bez podziału, } t=300^{\circ} \mathrm{C} \text {, gaz } \\
\text { nośny He, stałe ciśnienie } 23 \mathrm{psi}\end{array}$ & $\begin{array}{l}\text { splitless mode, } \mathrm{t}=250^{\circ} \mathrm{C} \text {, carrier gas } \\
\text { He, constant flow } 2 \mathrm{ml} / \mathrm{min} / \operatorname{tryb~bez} \\
\text { podziatu, } t=250{ }^{\circ} \mathrm{C}, \mathrm{gaz} \text { nośny } \mathrm{He}, \\
\text { staty przeptyw } 2 \mathrm{ml} / \mathrm{min}\end{array}$ \\
\hline $\begin{array}{l}\text { Temperature program/ } \\
\text { program temperaturowy }\end{array}$ & $\begin{array}{c}40^{\circ} \mathrm{C}(2 \mathrm{~min}) ; 3{ }^{\circ} \mathrm{C} / \mathrm{min}, 92^{\circ} \mathrm{C} \\
5^{\circ} \mathrm{C} / \mathrm{min}, 160{ }^{\circ} \mathrm{C} ; 10^{\circ} \mathrm{C} / \mathrm{min} \\
290^{\circ} \mathrm{C}(12 \mathrm{~min})\end{array}$ & $\begin{array}{c}40{ }^{\circ} \mathrm{C}(2 \mathrm{~min}) ; 3{ }^{\circ} \mathrm{C} / \mathrm{min}, 92{ }^{\circ} \mathrm{C} \\
5{ }^{\circ} \mathrm{C} / \mathrm{min}, 160{ }^{\circ} \mathrm{C} ; 10{ }^{\circ} \mathrm{C} / \mathrm{min} \\
290{ }^{\circ} \mathrm{C}(12 \mathrm{~min})\end{array}$ & $\begin{array}{c}80{ }^{\circ} \mathrm{C}(1 \mathrm{~min}) ; 10^{\circ} \mathrm{C} / \mathrm{min}, \\
100{ }^{\circ} \mathrm{C}(3 \mathrm{~min}) ; 30{ }^{\circ} \mathrm{C} / \mathrm{min}, \\
260^{\circ} \mathrm{C}(5 \mathrm{~min})\end{array}$ \\
\hline $\begin{array}{l}\text { Detector: FID MSD/ } \\
\text { Detektor:FID MSD }\end{array}$ & $\begin{array}{c}\mathrm{t}=350{ }^{\circ} \mathrm{C}, \mathrm{SCAN} \text { mode, mass range } \\
29-350[\mathrm{u}] / t=350^{\circ} \mathrm{C} \text { tryb } S C A N, \\
\text { zakres mas } 29-350[\mathrm{u}]\end{array}$ & $\begin{array}{c}t=350^{\circ} \mathrm{C} S C A N \text { mode, mass range } \\
29-260[u] / t=350{ }^{\circ} \mathrm{C} \text { tryb } S C A N, \\
\text { zakres mas } 29-260[u]\end{array}$ & $\begin{array}{c}\mathrm{t}=280{ }^{\circ} \mathrm{C}, \mathrm{SCAN} \text { mode, } \\
\text { mass range } 35-400[\mathrm{u}] / t=280^{\circ} \mathrm{C} \\
\text { tryb } S C A N, \text { zakres mas } 35-400[\mathrm{u}]\end{array}$ \\
\hline
\end{tabular}

In the case of hydrocarbon analysis, the sorbent tubes were subjected to thermal desorption (Markes UNITY2 thermodesorber, cold trap U-T11GPC-2S general purpose carbon $(\mathrm{C} 4 / 5-\mathrm{C} 30 / 32))$ for $15 \mathrm{~min}$ at a temperature of $300{ }^{\circ} \mathrm{C}$, followed by injection onto a chromatography column with a $30: 1$ split.

The sorbent tubes for aldehyde analysis were extracted using $1 \mathrm{ml}$ of toluene (with the addition of dimethylformamide (DMF) as an internal standard, $20 \mu \mathrm{l} \mathrm{DMF} / 100 \mathrm{ml}$ toluene), in ultrasonic bath for $30 \mathrm{~min}$. The parameters of the chromatography analysis are presented in Table 2.

The identification of compounds was performed based on the chromatogram obtained from the mass detector. The mass spectra of compounds were compared with NIST08 base spectra using the PBM algorithm (probability-based
Widma masowe związków porównywano z bazą widm NIST08, stosując algorytm PBM (probability-based matching). Związki z dopasowaniem powyżej $80 \%$ uznano za zidentyfikowane.

Analizę ilościową aldehydów przeprowadzono na podstawie wyników uzyskanych z detektora FID na podstawie krzywych wzorcowych poszczególnych związków.

\section{Analiza wyników badań}

\subsection{Paliwa badawcze}

Zgodnie ze świadectwem jakości mieszanek badawczych benzyna-etanol zawartość etanolu wynosiła odpowiednio: $\mathrm{E} 0<0,1 \%$; E5 $=5,0 \%$; E10 $=10,8 \%$; E25 = 26,4\%; E50 $=$ $=49,8 \%(\mathrm{v} / \mathrm{v})$. Porównawcza analiza chromatograficzna 
matching). Compounds with an $80 \%$ match (or better) were recognized as positively identified. Quantitative analysis of the aldehydes was conducted on the basis of results obtained from the FID detector, based on the calibration curves of individual compounds.

\section{Results and discussion}

\subsection{Fuels}

In accordance with the certificate for the gasoline-ethanol blends, the content of alcohol is, respectively: $\mathrm{E} 0<0.1 \%, \mathrm{E} 5=5.0 \%, \mathrm{E} 10=10.8 \%, \mathrm{E} 25=$ $=26.4 \%, \mathrm{E} 50=49.8 \%(\mathrm{v} / \mathrm{v})$. Comparative chromatographic analysis showed that there were no significant differences in the qualitative composition of the fuel blend. With the increase of ethanol concentration in the blend, compounds from gasoline decrease. A sample comparison of chromatograms for the E10 and E50 blends is shown in Fig. 4.

\subsection{Hydrocarbon analysis}

The main aim of the analysis was identification of hydrocarbons present in the exhaust gas. For this purpose, the exhaust gas was collected onto two different, complementary sorbents: Carbograph 1TD and Tenax TA. Carbograph 1TD belongs to the group of carbon sorbents, with a medium bond strength, and is used to analyze a wide range of volatile organic compounds. It is hydrophobic and does not demonstrate a tendency to create artifacts (i.e.

Abundance

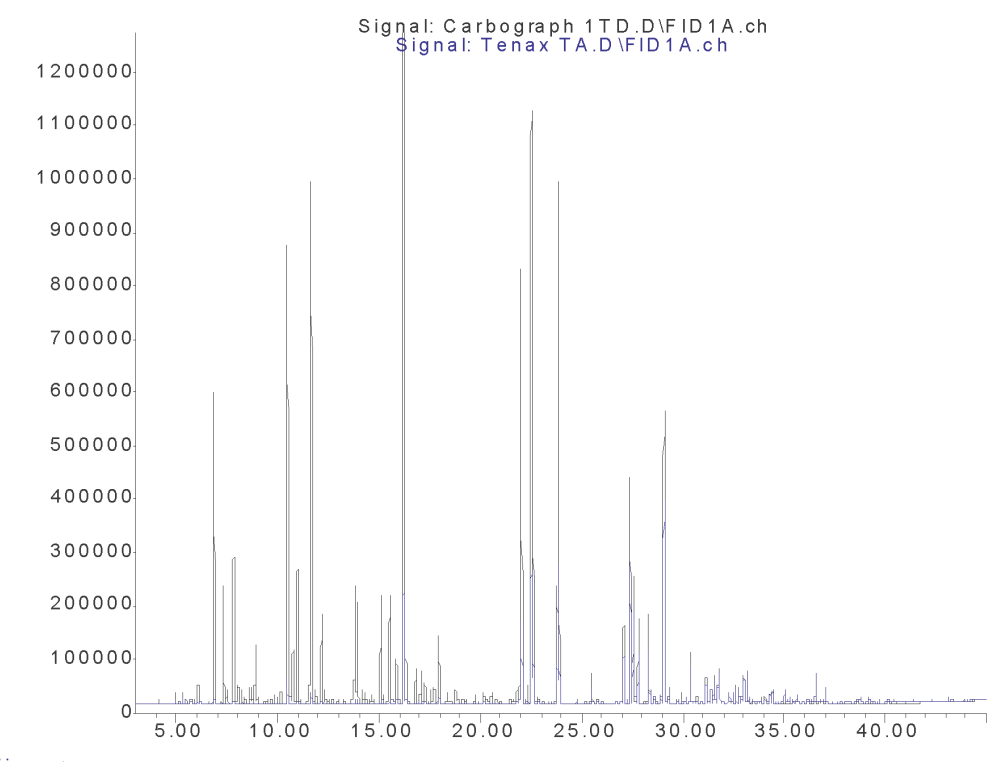

Fig. 5. Sample chromatograms of exhaust gases from the E0 fuel collected onto Carbograph 1TD and Tenax TA sorbents

Rys. 5. Przykładowe chromatogramy spalin z paliwa E0 pobrane na sorbenty Carborgaph ITD $i$ Tenax TA wykazała, że nie występują istotne różnice w składzie jakościowym mieszanki paliwowej. Ze wzrostem stężenia etanolu w mieszance maleje udział związków pochodzących z benzyny. Przykładowy fragment chromatogramów paliwa E10 i E50 przedstawiono na rys. 4.

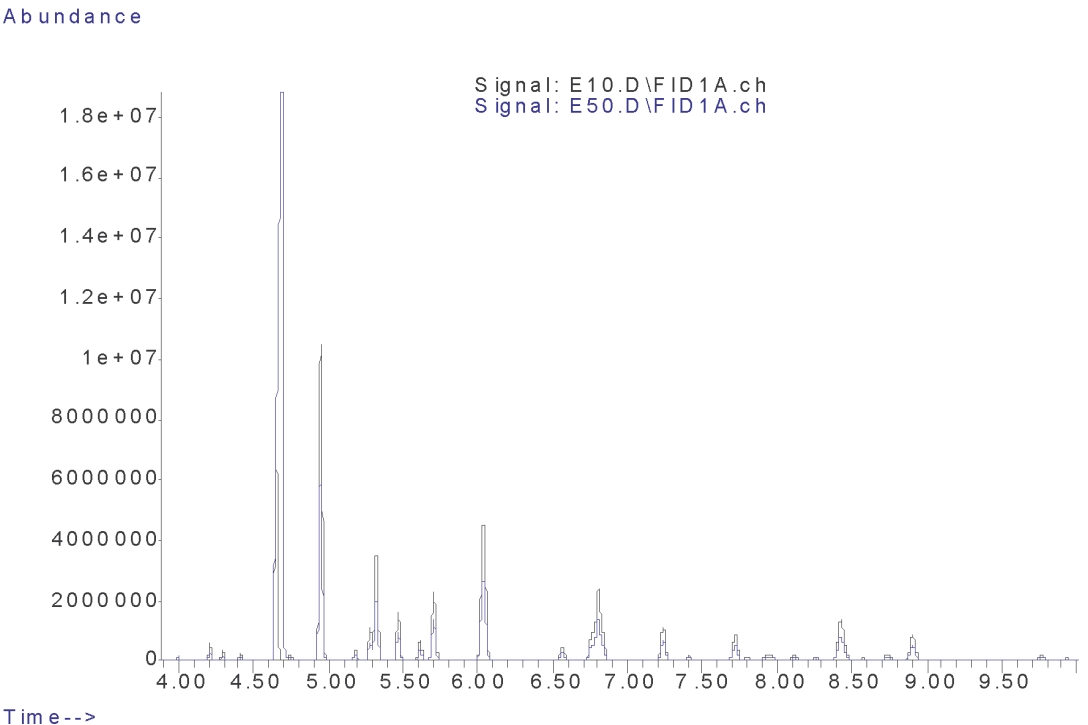

Fig. 4. Sample comparison of chromatograms for the E10 and E50 fuel blends; peak at retention time 4.70 min corresponds to the ethanol

Rys. 4. Fragmenty chromatogramów paliwa E10 i E50; pik przy czasie retencji 4,70 min odpowiada etanolowi

\subsection{Analiza węglowodorów}

Analiza węglowodorów obecnych w spalinach koncentrowała się na ich identyfikacji. W tym celu próbki spalin pobierano na dwa różne, uzupełniające się sorbenty: Carbograph 1TD oraz Tenax TA.

Carbograph 1TD należy do grupy sorbentów węglowych o średniej sile wiązania; używany jest do analizy szerokiego zakresu lotnych związków organicznych. Jest hydrofobowy i nie wykazuje skłonności do tworzenia artefaktów, czyli fałszywych pików (zakłóceń) na chromatogramach. Tenax TA jest hydrofobowym sorbentem polimerowym. Wykazuje skłonność do tworzenia artefaktów w kontakcie m.in. z ozonem, tlenkami azotu i innymi związkami utleniającymi obecnymi w próbce $[10,11]$. $Z$ tego względu przed złożem Tenax TA zastosowano filtry wstępne z tiosiarczanu sodu $\mathrm{Na}_{2} \mathrm{~S}_{2} \mathrm{O}_{3}$ oraz z jodku potasu KI. Analogicznie postępowano $\mathrm{W}$ przypadku poboru próbek na 2-HMP.

Przeprowadzone analizy spalin potwierdziły, że sorbenty uzupełniają się. Można zauważyć, że na sorbencie Carbograph 1TD lepiej niż na Tenax TA zatrzymywane są bardziej lotne związki organiczne (o czasie retencji do około $25 \mathrm{~min}$ ). Tenax TA zatrzymuje związki o wyższych temperaturach wrzenia, przez co 
false peaks (disturbances) on the chromatograms). Tenax TA is a hydrophobic polymer sorbent. It tends to create artifacts in contact with ozone, nitrogen oxides and other oxidizing compounds present in the sample $[10,11]$. Therefore, before the Tenax TA bed, pre-filters with $\mathrm{Na}_{2} \mathrm{~S}_{2} \mathrm{O}_{3}$ sodium thiosulfate and potassium iodide KI were used. An analogous procedure was undertaken while sampling onto 2-HMP. umożliwia dokładniejszą ich identyfikację. Przykładowe chromatogramy spalin z paliwa E0 przedstawiono na rys. 5 .

W wyniku analiz spalin z mieszanek benzyna-etanol pobieranych na powyższe sorbenty stwierdzono obecność około 250 związków, z których zidentyfikowano 126. Związki te podzielono na trzy podstawowe grupy, a uzyskane wyniki przedstawiono w tabeli 3 .

Table 3. Hydrocarbons identified in the exhaust gas

Tabela 3. Podzial węglowodorów zidentyfikowanych $w$ spalinach

\begin{tabular}{|l|c|c|c|}
\hline \multirow{2}{*}{} & \multicolumn{2}{|c|}{$\begin{array}{c}\text { Hydrocarbons identified in the exhaust gas/węglowodory zidentyfikowane } \\
\text { w spalinach }\end{array}$} \\
\cline { 2 - 4 } & Chains/tańcuchowe & \multicolumn{2}{|c|}{ Aliphatic/alifatyczne } \\
\cline { 2 - 4 } & 42 & 35 & 49 \\
\hline $\begin{array}{l}\text { Number of identified hydrocarbons/ } \\
\text { liczlentyfikowanych weglowodorów }\end{array}$ & & & \\
\hline
\end{tabular}

It can be seen that more volatile organic compounds (retention time up to $25 \mathrm{~min}$.) are retained on the Carborgraph 1TD sorbent, better than on Tenax TA (Fig. 5). Tenax TA retains compounds with higher boiling points, which enables more precise identification. Sample chromatograms with E0 fuel are shown in Figure 5.

As a result of the analysis of exhaust gas from the combustion gasoline-ethanol blends collected on the sorbents, about 250 compounds were found, of which 126 were identified. Those compounds were divided into three basic groups. The results are presented in Table 3.

Straight chain hydrocarbons $\mathrm{C} 5-\mathrm{C} 10$ and their branched derivatives were mainly identified among chain aliphatic hydrocarbons. The cyclic hydrocarbons identified are derivatives of cyclopropane, cyclopentane and cyclohexane.

A large group of compounds present in the exhaust gases were aromatic hydrocarbons. Compounds hazardous to human health and the environment were identified, including benzene, toluene, ethylbenzene, ethyltoluene, xylenes, styrene, cumene, as well as benzene derivatives (containing both straight and branched hydrocarbon chains as ligands).

Both aliphatic and aromatic hydrocarbons present in the exhaust gases can come from incompletely combusted fuel and result from complex reactions occurring in the engine's combustion chamber.

Ethanol present in the fuel blend is not completely burned during the combustion process in the engine, as evidenced by its presence in the exhaust gas. The products of incomplete oxidation of ethanol are acetaldehyde and acetic acid, which were also found in the exhaust gas.

\subsection{Aldehyde analysis}

During the collection of exhaust gases onto sorbent bed with 2-HMP,reactions leading to the formation of aldehyde derivatives occur, thus enabling analysis by gas chromatography. A schematic reaction of aldehydes with 2-HMP is shown in Fig. $6[12,13]$
Wśród łańcuchowych węglowodorów alifatycznych zidentyfikowano głównie proste węglowodory C5-C10 oraz ich rozgałęzione pochodne. Zidentyfikowane węglowodory cykliczne to pochodne cyklopropanu, cyklopentanu, cykloheksanu.

Dużą grupę związków obecnych w spalinach stanowią węglowodory aromatyczne. Zidentyfikowano związki niebezpieczne dla środowiska i zdrowia człowieka, takie jak benzen, toluen, etylobenzen, etylotoluen, ksyleny, styren, kumen, a także pochodne benzenu (zawierające jako podstawniki proste i rozgałęzione łańcuchy węglowodorowe).

Węglowodory zarówno alifatyczne, jak i aromatyczne obecne w spalinach mogą pochodzić z niecałkowicie spalonego paliwa oraz powstawać w wyniku złożonych reakcji zachodzących w komorze spalania silnika. Etanol obecny w paliwie nie jest całkowicie spalany w komorze silnika, o czym świadczy jego obecność w spalinach. Produktami niecałkowitego utleniania etanolu są obecne w spalinach acetaldehyd oraz kwas octowy.

\subsection{Analiza aldehydów}

Podczas poboru próbki na złożu z 2-HMP zachodzą reakcje prowadzące do powstawania pochodnych aldehydów, przez co możliwa jest ich analiza z wykorzystaniem chromatografii gazowej. Schematyczną reakcję powstawania pochodnych aldehydów z 2-HMP przedstawiono na rys. 6 $[12,13]$.

Przed rurką z sorbentem zastosowano filtry wstępne: jeden $z$ tiosiarczanu sodu $\mathrm{Na}_{2} \mathrm{~S}_{2} \mathrm{O}_{3}$, drugi z jodku potasu KI. Filtry mają za zadanie ograniczenie niekorzystnego wpływu tlenków azotu i ozonu obecnego w spalinach na sorbent. Gazy te powodują m.in. degradację złoża sorbentu, ponadto obecność ozonu obniża trwałości pochodnych aldehydów, co skutkuje zaniżaniem wyników analiz.

W trakcie analiz ślepej próby na sorbencie 2-HMP stwierdzono obecność acetaldehydu i formaldehydu, co uwzględniono na etapie przygotowania metody analitycznej. 
<smiles>OCC1CCCCN1</smiles><smiles>[R]C=O</smiles>

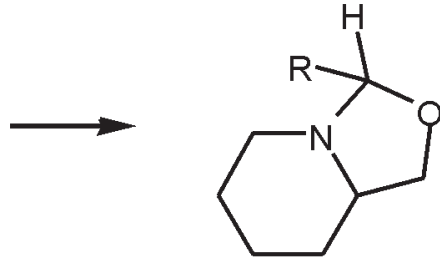

Fig. 6. Schematic reaction of aldehydes with 2-HMP

Rys. 6. Schemat reakcji 2-HMP z aldehydami
Analiza spalin wykazała obecność acetaldehydu oraz śladowych ilości formaldehydu i benzaldehydu, niepozwalających na ich oznaczenie ilościowe. Stosując opisaną metodę analityczną, nie stwierdzono obecności innych aldehydów. Może to być

Pre-filters, with sodium thiosulfate $\mathrm{Na}_{2} \mathrm{~S}_{2} \mathrm{O}_{3}$ and potassium iodide KI, were applied in front of the sorbent tube. Filters were used to reduce the negative effect of nitrogen oxides and ozone in the exhaust gases to the sorbent. These gases cause, among other effects, degradation of the sorbent bed. Moreover, the presence of ozone reduces the stability of aldehyde derivatives, leading to underestimation of the derivative concentration. During the analysis of a blank test of 2-HMP, acetaldehyde and formaldehyde were found, which was considered in the analytical method.

Analysis of exhaust gases revealed the presence of acetaldehyde and trace amounts of formaldehyde and ben- spowodowane ograniczeniami metody lub małymi stężeniami tych aldehydów w spalinach z mieszanek benzyna-etanol.

Zawartość acetaldehydu w spalinach pozwala na jego oznaczenie ilościowe. W tabeli 4 przedstawiono zawartość acetaldehydu w spalinach w zależności od stężenia etanolu w paliwie. Wynik analizy chromatograficznej został przeliczony na emisję spalin $\left(5,574 \mathrm{~m}^{3}\right)$ wyrażoną $\mathrm{w} \mathrm{m}^{3}$ oraz na teoretyczną drogę przebytą przez samochód w teście $(11,007$ $\mathrm{km}$ ) wyrażoną w km. Zależność stężenia acetaldehydu w spalinach od stężenia etanolu w paliwie wraz z zakresem błędu RSD przedstawiono graficznie na rys. 7.

Table 4. Acetaldehyde emission as a function of the concentration of ethanol in the blend

Tabela 4. Emisja acetaldehydu $w$ spalinach $w$ zależności od stężenia etanolu $w$ paliwie

\begin{tabular}{|l|c|c|c|c|}
\hline \multirow{2}{*}{ Fuel/paliwo } & $\begin{array}{c}\text { EtOH content [\%]/ } \\
\text { zawartośc EtOH [\%] }\end{array}$ & $\begin{array}{c}\text { concentration of acetaldehyde in } \\
\text { exhaust gases/stęzenie acetalde- } \\
\text { hydu } w \text { spalinach }\end{array}$ & $\begin{array}{c}\text { acetaldehyde road emis- } \\
\text { sion/emisja drogowa } \\
\text { acetaldehydu }\end{array}$ \\
\cline { 2 - 4 } & & {$\left[\mu \mathrm{g} / \mathrm{m}^{3}\right]$} & {$[\mu \mathrm{g} / \mathrm{km}]$} \\
\hline E0 & $<0.1$ & 2.10 & 0.57 \\
\hline E5 & 5.0 & 4.81 & 1.31 \\
\hline E10 & 10.8 & 9.35 & 2.55 \\
\hline E25 & 26.4 & 15.07 & 4.11 \\
\hline E50 & 49.8 & 21.84 & 3.13 \\
\hline RSD [\%] relative standard deviation (based on three measurements)/RSD [\%] względne odchylenie standardowe (z trzech pomiarów) & 4.85 \\
\hline
\end{tabular}

zaldehyde, which did not permit their quantification. Using the method of analysis described, other aldehydes were not detected. This may be due to the limitations of the method or low concentrations of these aldehydes in the exhaust gases generated from gasoline-ethanol blends.

The content of acetaldehyde in the exhaust gas permits its quantification. Table 4 shows the acetaldehyde content in the exhaust gas depending on the concentration of ethanol in the fuel. The result of chromatographic analysis has been recalculated, based on total exhaust emissions $\left(5.574 \mathrm{~m}^{3}\right)$, expressed in $\mathrm{m}^{3}$, and the theoretical distance traveled by the car during the test $(11.007 \mathrm{~km})$, expressed in $\mathrm{km}$.

Dependence of concentration of acetaldehyde on ethanol content in the fuel blends with RSD error are shown graphically in Figure 7.

Based on these data, it was found that the concentration of acetaldehyde in the exhaust gas depends largely on the ethanol content in the fuel blend. Increasing the concentration of ethanol from $5 \%$ (base fuel) to $10 \%$ resulted in twice the
Na podstawie powyższych danych można stwierdzić, że stężenie acetaldehydu w spalinach zależy w dużym stopniu od zawartości etanolu w mieszance paliwowej. Wzrost stężenia etanolu z 5\% (paliwo bazowe) do 10\% spowodował dwukrotnie wyższą emisję acetaldehydu. Dalsze zwiększanie zawartości etanolu w paliwie nie powoduje tak gwałtownego przyrostu stężenia tego aldehydu w spalinach. W porównaniu do E5, spaliny z E25 zawierają nieco ponad 3-krotnie więcej, a z E50 około 4,5-krotnie więcej acetaldehydu.

Obecność acetaldehydu w spalinach z E0 potwierdza, że nie powstaje on tylko na skutek niecałkowitego utleniania etanolu, ale jest również produktem procesów spalania węglowodorów obecnych w benzynie.

Wartość względnego odchylenia standardowego (RSD), które za wyjątkiem paliwa E0 wynosi poniżej 5\%, wskazuje na dobrą powtarzalność analiz, potwierdzając dobrą jakość metody analitycznej wykorzystywanej w badaniach. Dla paliwa E0 większa wartość RSD może 
emissions of acetaldehyde. However, further increases of the ethanol content in the fuel does not cause such a rapid increase in the concentration of the aldehyde in the exhaust. Compared to E5, E25 exhaust gases contain just over 3 times more acetaldehyde, and in case of E50 about 4.5 times more.

The presence of acetaldehyde in the exhaust gases from E0 confirms that it is not only formed due to incomplete oxidation of ethanol, but is also a product of combustion of hydrocarbons present in gasoline.

The value of relative standard deviation (RSD), which except for the E0 fuel,

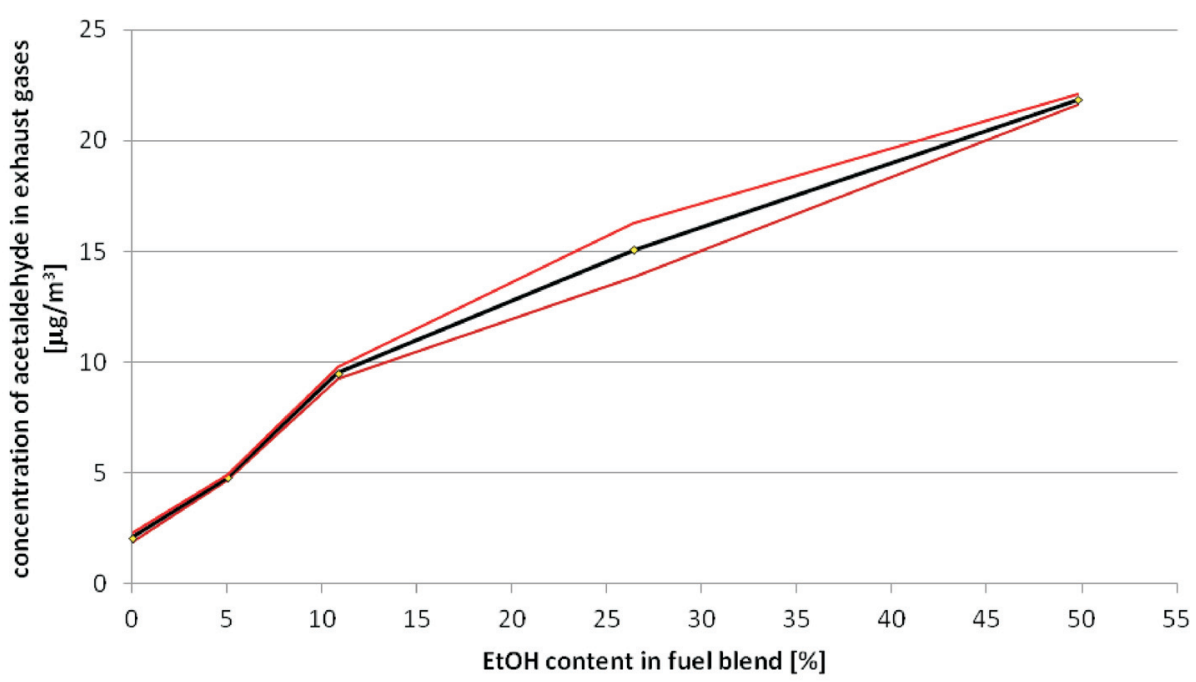
less than $5 \%$, indicating good reproducibility of the analysis, confirming the quality of the analytical method used in the study. In the case of E0 fuel, the higher RSD value may be due to the relatively low content of acetaldehyde in the exhaust gases.

The low content of formaldehyde detected in the exhaust gas may be a result of imperfections in the method - intermediate stage of sampling into the Tedlar bag. This intermediate stage of sampling can lead to an aldehyde concentration decrease, due to the high reactivity of carbonyl compounds.

Probably the modification of sampling stages, skipping the Tedlar bag collection step, and sampling the exhaust gas directly from the tailpipe onto the sorbent, could allow detection of large amounts of formaldehyde and/or other aldehydes. Despite its limitations, the method has proved useful for the determination of acetaldehyde in the exhaust gases.

\section{Conclusions}

Application of thermaldesorption and gas chromatography analysis GC/MS allows the identification of a large number of hydrocarbons present in the exhaust gases generated from gasoline-ethanol blends. Using the sampling of different, complementary sorbents, such as Carbograph 1TD and Tenax TA, allows for more exact identification of the large number of compounds. Nearly half of the identified hydrocarbons are aromatic compounds, hazardous to health, including benzene, toluene, xylenes, styrene and ethylbenzene, among others.

Sampling of exhaust gas on a bed of XAD-2 coated with 2-HMP and followed by GC-MS/FID analysis revealed the presence of acetaldehyde, and trace amounts of formaldehyde and benzaldehyde. The amount of acetaldehyde permitted quantitative analysis.

It was found that an increase of ethanol concentration in the fuel blend lead to an increase in acetaldehyde concen-

być wynikiem niewielkiej zawartości acetaldehydu w spalinach.

Mała zawartość formaldehydu w spalinach, uniemożliwiająca oznaczenie ilościowe, może świadczyć o niedoskonałości metody - pośredni etap poboru do worka Tedlar. Etap pośredni poboru może prowadzić do zmniejszenia stężenia aldehydów ze względu na dużą reaktywność związków karbonylowych.

Prawdopodobnie modyfikacja etapu poboru próbek (pominięcie etapu poboru do worka Tedlar, pobór bezpośrednio z rury wylotowej na sorbent) mogłaby pozwolić na analizę ilościową emisji formaldehydu i ewentualnie innych aldehydów. Pomimo swoich ograniczeń metoda okazała się użyteczna do oznaczania zawartości acetaldehydu w spalinach.

\section{Wnioski}

Zastosowanie techniki termodesorpcji i analizy chromatograficznej GC/MS pozwala na identyfikację bardzo dużej liczby węglowodorów obecnych w spalinach generowanych z mieszanek benzyna-etanol. Wykorzystanie do poboru spalin odmiennych, uzupełniających się sorbentów, jakimi są Carbograph 1TD i Tenax TA, pozwala na dokładniejszą identyfikację większej liczby związków. Ze zidentyfikowanych węglowodorów prawie połowa to niebezpieczne dla zdrowia związki aromatyczne, m. in. benzen, toluen, ksyleny, styren, etylobenzen.

Pobór próbek spalin na złoże XAD-2 pokryte 2-HMP, a następnie analiza GC-MS/FID wykazały obecność acetaldehydu oraz śladowych ilości formaldehydu i benzaldehydu. Zawartość acetaldehydu pozwoliła na jego analizę ilościową. Stwierdzono, że ze wzrostem stężenia etanolu $\mathrm{w}$ mieszance paliwowej wzrasta stężenia acetaldehydu $\mathrm{w}$ spalinach. Wzrost stężenia etanolu z 5\% (paliwo bazowe) do $10 \%$ powoduje dwukrotny wzrost stężenia acetaldehy- 
tration in the exhaust gas. Increasing the concentration of ethanol from $5 \%$ (base fuel) to $10 \%$ will double the emission of acetaldehyde. In the case of the $50 \%$ ethanol blend, emission of acetaldehyde is 4.5 times greater than in case of the base (5\% ethanol) fuel blend.

\section{Acknowledgements}

The authors acknowledge the financial support of the Polish Ministry of Science and Higher Education (Project N N509 494 838) funds for years 2010-2012. du. $\mathrm{W}$ odniesieniu do mieszanki zawierającej 50\% etanolu emisja acetaldehydu jest 4,5-krotnie większa niż dla paliwa bazowego (mieszanki zawierającej 5\% etanolu).

\section{Podziękowania}

Badania zostały wykonane w ramach projektu badawczego finansowanego przez Ministerstwo Nauki i Szkolnictwa Wyższego (Projekt N N509 494838) w latach 2010-2012.

Paper reviewed/Artykuł recenzowany

\section{Bibliography/Literatura}

[1] Pang X., Shi X., Mu Y., He H., Shuai S., Chen H., Li, R.: Characteristics of carbonyl compounds emission from a dieselengine using biodiesel-ethanol-diesel as fuel. Atmospheric Environment, 40, 7057-7065, 2006.

[2] Commission Regulation (EC) No 692/2008 of 18 July 2008 implementing and amending Regulation (EC) No 715/2007 of the European Parliament and of the Council on type-approval of motor vehicles with respect to emissions from light passenger and commercial vehicles (Euro 5 and Euro 6) and on access to vehicle repair and maintenance information. Official Journal of the European Union, L199, 1-136, 28.7.2008.

[3] Bielaczyc P., Szczotka A., Woodburn J.: A Study of Gasoline-Ethanol Blends Influence on Performance and Exhaust Emission from a Light-Duty Gasoline Engine, SAE Technical Paper 2012-01-1052.

[4] Bielaczyc P., Szczotka A., Woodburn J.: The Effect of Various Petrol-Ethanol Blends on Exhaust Emission and Fuel Consumption of an Unmodified Light-Duty SI Vehicle, SAE Technical Paper 2011-24-0177.

[5] Magnusson R., Nilsson C.: The influence of oxygenated fuels on emissions of aldehydes and ketones from a two-stroke spark ignition engine. Fuel, Vol. 90, 1145-1154, 2011.

[6] Lemaire R., Therssen E., Desgroux P.: Effect of ethanol addition in gasoline and gasoline-surrogate on soot formation in turbulent spray flames. Fuel, Vol. 89, Issue 12, 3952-3959, 2010.

[7] Jia L.W., Shen M.Q., Wang J., Lin M.Q.: Influence of ethanol-gasoline blended fuel on emission characteristics from a four-stroke motorcycle engine. Journal of Hazardous Materials, A123, 29-34, 2005.

[8] Bielaczyc P., Pajdowski P., Szczotka A., Woodburn J.: Development of vehicle exhaust emission testing methods - BOSMAL's new emission testing laboratory. PTNSS-2011SS1-101, Combustion Engines, 1/2011.

[9] Directive 98/69/EC of the European Parliament and of the Council of 13 October 1998. 1998L0069, 1998.

[10] Clausen P.A., Wolkoff P.: Degradation products of Tenax TA formed during sampling and thermal desorption analysis: indicators of reactive species indoors. Atmospheric Environment, Vol. 31, No. 5, 715-725, 1997.

[11] Lee J.H., Batterman S.A., Jia, C., Chernyak S.: Ozone Artifacts and Carbonyl Measurements Using Tenax GR, Tenax TA, Carbopack B, and Carbopack X Adsorbents, Journal Air \& Waste Manage. Assoc., Vol. 56, 1503-1517, 2006.

[12] NIOSH Manual of Analytical Methods, Methods 2501, Issue 2, 15 August 1994.

[13] NIOSH Manual of Analytical Methods, Methods 2539, Issue 2, 15 August 1994.

Damian Łomankiewicz, MSc. - chemical laboratory manager, BOSMAL Automotive Research and Development Institute Ltd in Bielsko-Biała.

Mgr Damian Łomankiewicz - kierownik Pracowni Chemicznej, Zakład Materiałoznawstwa, Instytut Badań i Rozwoju Motoryzacji BOSMAL Sp. z o.o., Bielsko-Biała. e-mail:damian.lomankiewicz@bosmal.com.pl

Krzysztof Brodzik, DEng. - research-technical specialist, BOSMAL Automotive Research and Development Institute Ltd in Bielsko-Biała.

Dr inż. Krzysztof Brodzik - specjalista badawczo-techniczny w Zakładzie Materiałoznawstwa, Instytut Badań $i$ Rozwoju Motoryzacji BOSMAL Sp. z o.o., Bielsko-Biała. e-mail: krzysztof.brodzik@bosmal.com.pl
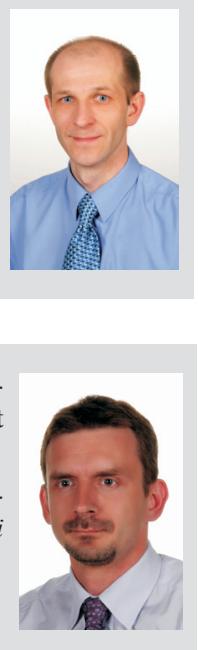

Piotr Bielaczyc, DEng. - head of the Engine Research Department, BOSMAL Automotive Research and Development Institute Ltd in Bielsko-Biała.

Dr inż. Piotr Bielaczyc - kierownik Zakładu Badań Silników, Instytut Badań i Rozwoju Motoryzacji BOSMAL Sp. z o.o., Bielsko-Biała. e-mail:piotr.bielaczyc@bosmal.com.pl

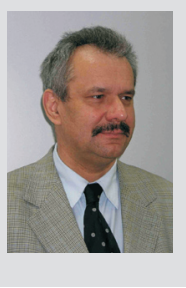

